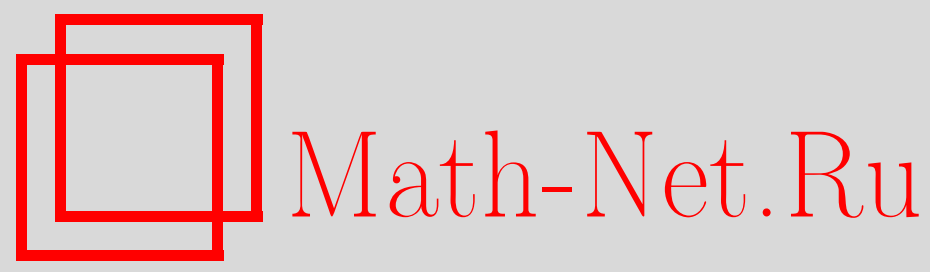

А. О. Иванов, А. А. Тужилин, Д. Цислик, Отношение Штейнера для многообразий, Матем. заметки, 2003, том 74, выпуск 3, 387-395

DOI: https://doi.org/10.4213/mzm272

Использование Общероссийского математического портала Math-Net.Ru подразумевает, что вы прочитали и согласны с пользовательским соглашением http://www.mathnet.ru/rus/agreement

Параметры загрузки:

IP : 54.237 .59 .107

26 апреля 2023 г., $17: 24: 42$

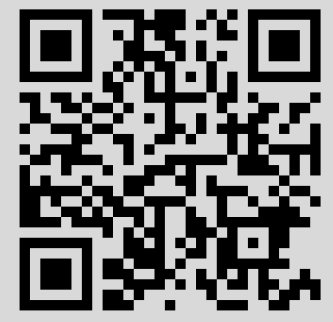




\title{
ОТНОШЕНИЕ ШТЕЙНЕРА ДЛЯ МНОГООБРАЗИЙ
}

\author{
А. О. Иванов, А.А. Тужилин, Д. Цислик
}

\begin{abstract}
Отношение Штейнера характеризует максимальное возможное отклонение длины минимального остовного дерева от длины минимального дерева Штейнера. В статье получены оценки на отношение Штейнера на римановых многообразиях. В качестве следствия вычислено отношение Штейнера для плоских торов, плоских бутылок Клейна и проективной плоскости постоянной положительной кривизны.

Библиограффия: 7 названий.
\end{abstract}

1. Введение. Пусть $V$ - произвольное конечное множество. Напомним, что графом $G$ на множестве $V$ называется пара $(V, E)$, где $E$ - некоторое конечное семейство пар элементов множества $V$. В семействе $E$ могут встречаться пары с одинаковыми элементами, а также одинаковые пары. Элементы из $V$ называются вершинами графа $G$,

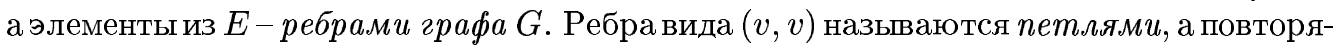
юшиеся пары из $E$ называются кратными ребрами. Если задан граф $G$, то множество его вершин обычно обозначается через $V(G)$, а множество его ребер - через $E(G)$. Для удобства мы будем часто обозначать ребро $e=\{x, y\} \in E(G)$ через $x y$.

Иногда бьвает удобно рассматривать граф как топологическое пространство, склеенное из отрезков, каждьй из которых соответствует некоторому ребру графа. Такие графы мы будем назьвать топологическими, а непрерьвное отображение топологичес-

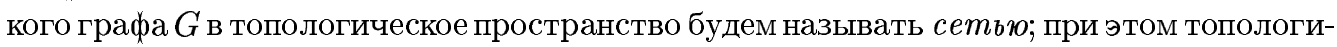
ческий граф $G$, а также обычньй граф, соответствуюший $G$, назовем типом этой сети или ее топологией. Таким образом, ребра сети - это непрерьвные кривые в объемлющем пространстве. Кроме того, вся терминология теории топологических пространств переносится на топологические графы и сети. Если объемлющее пространство является гладким многообразием, то сеть в таком пространстве назовем гладкой (кусочно-гладкой), если все ребра сети являются гладкими (кусочно-гладкими).

Граф $G$ назьвается взвешенным , если на множестве его ребер задана неотрицательная функция $\omega: E(G) \rightarrow \mathbb{R}$, называемая весовой функиией. При этом число $\omega(e)$ называется весом ребра $е \in E(G)$. Сумма весов всех ребер взвешенного графа $G$ назьвается весом графа $G$ и обозначается через $\omega(G)$. Если $G$ - связньй взвешенньй граф, то из всех связных остовных подграфов в $G$ наименьшего веса всегда можно выбрать дерево,

При подготовке данной работы А. О. Иванов и А. А. Тужилин пользовались частичной поддержкой Российского фонда фундаментальных исследований, гранты №№ 96-15-96142, 98-01-00240, и INTAS, проект № 97-0808. 
которое называется минимальным остовным деревом и обозначается через $\mathrm{MST}_{G}$. Отметим, что если все веса строго больше нуля, то любой связньй остовный подграфф в $G$ наименьшего веса является деревом.

Пусть $X$-множество, $\rho$ - некоторая метрика на $X$, и $N$-произвольное конечное подмножество в $X$. Пусть $G$-полный граф на множестве $N$. Метрика $\rho$ порождает весовую функцию, ставящую в соответствие каждому ребру $x y \in E(G)$ число $\rho(x, y)$. Эту весовую функцию мы будем обозначать той же буквой $\rho$. Минимальное взвешенное дерево во взвешенном графе $G$ обозначим через $\mathrm{MST}_{N}$. Минимальным деревом Штейнера на множсестве $N$ или минимальным деревом Штейнера, затягиваюшим множество $N$, назьвается дерево $\Gamma, N \subset V(\Gamma)$, для которого

$$
\rho(\Gamma)=\inf _{\left.\left\{\bar{N}: \bar{N}_{\subset}\right\}\right\}} \rho\left(\mathrm{MST}_{\bar{N}}\right),
$$

где точная нижняя грань берется по всем конечньп подмножествам $\bar{N}$ в $X$, содержащим $N$. Минимальное дерево Штейнера на множестве $N$ обозначается через $\mathrm{SMT}_{N}$.

Отметим, что, вообще говоря, не для всякого $N$ существует $\mathrm{SMT}_{N}$ (причиной этого может служить неполнота метрического пространства $(X, \rho))$. Тем не менее, сама точная нижняя грань из определения $\mathrm{SMT}_{N}$ существует всегда. $B$ дальнейшем, дажсе если $\mathrm{SMT}_{N}$ не существует, точную нижнюю грань (1) будем обозначать через $\rho\left(\mathrm{SMT}_{N}\right)$.

ОПРЕДЕЛЕНИЕ. Отношением Штейнера $m(X, \rho)$ метрического пространства $(X, \rho)$ назьвается следующая величина:

$$
m(X, \rho)=\inf _{\{N: N \subset X\}} \frac{\rho\left(\mathrm{SMT}_{N}\right)}{\rho\left(\mathrm{MST}_{N}\right)} .
$$

Ясно, что $0 \leqslant m(X, \rho) \leqslant 1$.

Приведем основные известные нам результаты, описьвающие отношение Штейнера римановых многообразий. Ду и Хванг [1] показали, что отношение Штейнера евклидовой плоскости равно $\sqrt{3} / 2$; тем самым, была доказана знаменитая гипотеза Гилберта и Поллака [2], не поддававшаяся решению в течение 30 лет. Имеется также ряд оценок на отношение Штейнера для общих евклидовых пространств $\mathbb{R}^{n}$, см. подробности в [3]. Рубинштейн и Венг [4] вычислили отношение Штейнера двумерной сферы постоянной (положительной) кривизны: оно оказалось таким же, как и у $\mathbb{R}^{2}$, т.е. равным $\sqrt{3} / 2$.

Используя технику, разработанную в [5] и [6], авторы настоящей статьи получили следуюшие результаты.

Теорема 1.1. Отношение Штейнера произвольного связного нова многообразия не превосходит отночения Штейнера евклидова пространства $\mathbb{R}^{n}$.

Теорема 1.2. Пусть $W$ и $M$ - связные римановы многообразия, $u \pi: W \rightarrow M-$ локально изометричное накрытие. Тогда отношение Штейнера базы $M$ не меньше отношения Штейнера тотального пространства $W$. 
СЛЕДСТВИЕ 1.1. Если связное риманово многообразие можнно локально изометрично накрыть евклидовым пространством $\mathbb{R}^{n}$, то отношение Штейнера этого многообразия равно отношению ШІтейнера пространства $\mathbb{R}^{n}$.

СлЕДСТВИЕ 1.2. Отношение Штейнера плоского двумерного тора, плоской бутылки Клейна, проективной плоскости постоянной положительной кривизнь равно $\sqrt{3} / 2$.

Таким образом, учитьвая результат Рубинштейна и Венга [4], отношение Штейнера вычислено для всех замкнутых двумерных поверхностей постоянной неотрицательной кривизны.

ТЕОрема 1.3. Отношение Штейнера пространства Лобачевского кривизны -1 не превосходит $3 / 4$.

ТЕОрема 1.4. Отношение Штейнера произвольной двумерной поверхности постоянной кривизны -1 строго меньие $\sqrt{3} / 2$.

А. О. Иванов и А.А. Тужилин пользуются случаем выразить искреннюю признательность А. Т. Фоменко за постоянное внимание к их работе. Авторы также благодарны университету г. Гряйфсвальд (Германия) за поддержку их встречи в Гряйфсвальде в марте 2000 г.

2. Доказательства теорем. В настоящем разделе мы приведем доказательства всех сформулированных во введении результатов. Для этого нам понадобятся следующие хорошо известные факты.

Доказательство приводимых ниже двух лемм см. в [3] (отметим, что лемма 2.1 доказана в [3] лишь для нормированных пространств, хотя ее доказательство для метрического случая абсолютно такое же).

ЛЕмма 2.1. Пусть $X$ - некоторое множество, а $\rho_{1}$ и $\rho_{2}-$ две метрики на множестве $X$. Предполохим, что для некоторых чисел $c_{2} \geqslant c_{1}>0$ и любых двух точек $x$ и у из $X$ выполняется $c_{1} \rho_{2}(x, y) \leqslant \rho_{1}(x, y) \leqslant c_{2} \rho_{2}(x, y)$. Тогда

$$
\frac{c_{1}}{c_{2}} m\left(X, \rho_{2}\right) \leqslant m\left(X, \rho_{1}\right) \leqslant \frac{c_{2}}{c_{1}} m\left(X, \rho_{2}\right) .
$$

ЛЕмма 2.2. Пусть $(X, \rho)$ - метрическое пространство, и $Y \subset X$ - его подпространство. Тогда

$$
m(Y, \rho) \geqslant m(X, \rho) .
$$

Воспользуемся этими леммами для доказательства следующего предложения.

ПРЕДЛОЖЕНИЕ 2.1. Пусть $f: X \rightarrow Y$ - отображение метрического пространства $\left(X, \rho_{X}\right)$ на метрическое пространство $\left(Y, \rho_{Y}\right)$, не увеличивающее расстояний, т.е. для любых двух точек $x$ и $у$ из $X$ выполняется

$$
\rho_{Y}(f(x), f(y)) \leqslant \rho_{X}(x, y) .
$$

Тогда для любого конечного множества $N \subset Y$ выполняется

$$
\rho_{X}\left(\operatorname{MST}_{N}\right) \geqslant \rho_{Y}\left(\operatorname{MST}_{f(N)}\right), \quad \rho_{X}\left(\operatorname{SMT}_{N}\right) \geqslant \rho_{Y}\left(\operatorname{SMT}_{f(N)}\right) .
$$


ДокАЗАТЕЛЬСтво. Пусть $G$-произвольный связньй граф, построенньй на $N$. Рассмотрим на $G$ две весовых функции, заданные на ребрах $x y$ из $G$ так: $\rho_{X}(x y)=\rho_{X}(x, y)$ и $\omega_{Y}(x y)=\rho_{Y}(f(x), f(y))$. Так как $f$ не увеличивает расстояний, то $\rho_{X}(G) \geqslant \omega_{Y}(G)$.

Пусть $G^{\prime}$ - граф на $N^{\prime}=f(N)$ такой, что вершины $x^{\prime}$ и $y^{\prime}$ из $N^{\prime}=V\left(G^{\prime}\right)$ соединены столькими ребрами, сколько ребер из $G$ соединяет вершины из $f^{-1}\left(x^{\prime}\right) \cap N$ с вершинами из $f^{-1}\left(y^{\prime}\right) \cap N$. Ясно, что $G^{\prime}-$ связньй граф такой, что $\rho_{Y}\left(G^{\prime}\right)=\omega_{Y}(G)$.

Обратно, легко видеть, что для любого связного графа $G^{\prime}$, построенного на $f(N)$, существует такой связньй граф $G_{X}$ на $N$, что $\rho_{Y}\left(G^{\prime}\right)=\omega_{Y}\left(G_{X}\right)$ (для построения графа $G_{X}$ достаточно затянуть связным графом каждое множество $N \cap f^{-1}\left(x^{\prime}\right), x^{\prime} \in N^{\prime}$, а каждую пару полученных связных графов, соответствующих смежным в $G^{\prime}$ вершинам, соединить столькими ребрами, какова кратность соответствующего ребра из $G^{\prime}$ ). Поэтому

$$
\begin{aligned}
\rho_{X}\left(\mathrm{MST}_{N}\right) & =\inf _{\{G: V(G)=N\}} \rho_{X}(G) \geqslant \inf _{\{G: V(G)=N\}} \omega_{Y}(G) \\
& =\inf _{\left\{G^{\prime}: V\left(G^{\prime}\right)=f(N)\right\}} \rho_{Y}\left(G^{\prime}\right)=\rho_{Y}\left(\operatorname{MST}_{f(N)}\right) .
\end{aligned}
$$

Тем самьм, первое неравенство доказано.

Докажем теперь второе неравенство. Имеем

$$
\begin{aligned}
\rho_{X}\left(\mathrm{SMT}_{N}\right) & =\inf _{\{\bar{N}: \bar{N} \supset N\}} \rho_{X}\left(\operatorname{MST}_{\bar{N}}\right) \geqslant \inf _{\{\bar{N}: \bar{N} \supset N\}} \rho_{Y}\left(\operatorname{MST}_{f(\bar{N})}\right) \\
& \geqslant \inf _{\left\{\bar{N}^{\prime}: \bar{N}^{\prime} \supset f(N)\right\}} \rho_{Y}\left(\mathrm{MST}_{\bar{N}^{\prime}}\right)=\rho_{Y}\left(\mathrm{SMT}_{f(N)}\right) .
\end{aligned}
$$

Доказательство закончено.

ПРЕДЛОЖЕНИЕ 2.2. Пусть $f: X \rightarrow Y$ - отображсение метрического пространства $\left(X, \rho_{X}\right)$ в метрическое пространство $\left(Y, \rho_{Y}\right)$, не увеличивающее расстояний. Предположим, что для каждого конечного подмножества $N^{\prime} \subset Y$ существует такое конечное подмнохсество $N$, что $f(N)=N^{\prime} u$

$$
\rho_{X}\left(\mathrm{SMT}_{N}\right) \leqslant \rho_{Y}\left(\mathrm{SMT}_{N^{\prime}}\right)
$$

Tогда

$$
m\left(X, \rho_{X}\right) \leqslant m\left(Y, \rho_{Y}\right)
$$

ДокАЗАТЕЛЬСтво. Пусть $N \subset X$ - произвольное конечное множество. Имеем

$$
\begin{aligned}
m\left(X, \rho_{X}\right) & =\inf _{\{N: N \subset X\}} \frac{\rho_{X}\left(\mathrm{SMT}_{N}\right)}{\rho_{X}\left(\mathrm{MST}_{N}\right)}=\inf _{\left\{N^{\prime}: N^{\prime} \subset Y\right\}\left\{N: f(N)=N^{\prime}\right\}} \frac{\rho_{X}\left(\mathrm{SMT}_{N}\right)}{\rho_{X}\left(\mathrm{MST}_{N}\right)} \\
& \leqslant \inf _{\left\{N^{\prime}: N^{\prime} \subset Y\right\}} \frac{\rho_{Y}\left(\mathrm{SMT}_{N^{\prime}}\right)}{\rho_{Y}\left(\mathrm{MST}_{N^{\prime}}\right)}=m\left(Y, \rho_{Y}\right),
\end{aligned}
$$

где неравенство получается одновременным применением условия (2) и первого неравенства из предложения 2.1. Доказательство закончено.

Предложение 2.2 можно немного усилить. 
ПрЕДЛОЖЕНИЕ 2.3. Пусть $f: X \rightarrow Y$ - отображение метрического пространства $\left(X, \rho_{X}\right)$ в метрическое пространство $\left(Y, \rho_{Y}\right)$, не увеличивающее расстояний. Предположим, что для каждого конечного подмножества $N^{\prime} \subset Y$ выполняется

$$
\inf _{\left\{N: f(N)=N^{\prime}\right\}} \rho_{X}\left(\mathrm{SMT}_{N}\right) \leqslant \rho_{Y}\left(\mathrm{SMT}_{N^{\prime}}\right) .
$$

Тогда

$$
m\left(X, \rho_{X}\right) \leqslant m\left(Y, \rho_{Y}\right)
$$

ДокАЗАТЕЛЬСтво. Пусть $N \subset X-$ произвольное конечное множество. Как и в доказательстве предложения 2.2 , имеем

$$
m\left(X, \rho_{X}\right)=\inf _{\{N: N \subset X\}} \frac{\rho_{X}\left(\mathrm{SMT}_{N}\right)}{\rho_{X}\left(\mathrm{MST}_{N}\right)}=\inf _{\left\{N^{\prime}: N^{\prime} \subset Y\right\}} \inf _{\left\{N: f(N)=N^{\prime}\right\}} \frac{\rho_{X}\left(\mathrm{SMT}_{N}\right)}{\rho_{X}\left(\mathrm{MST}_{N}\right)} .
$$

Так как $f$ не увеличивает расстояний, то $\rho_{X}\left(\mathrm{MST}_{N}\right) \geqslant \rho_{Y}\left(\mathrm{MST}_{f(N)}\right)$ (см. предложение 2.1); с другой стороны, по условию существует такая последовательность конечных множеств $N_{i} \subset X, f\left(N_{i}\right)=N^{\prime}$, что $\rho_{X}\left(\mathrm{SMT}_{N_{i}}\right) \leqslant \rho_{Y}\left(\mathrm{SMT}_{N^{\prime}}\right)+\varepsilon_{i}$, где последовательность положительных чисел $\varepsilon_{i}$ стремится к 0 при $i \rightarrow \infty$, а последовательность положительных чисел $\rho_{X}\left(\mathrm{SMT}_{N_{i}}\right)$ стремится к $\left.\inf _{\{N}: f(N)=N^{\prime}\right\} \rho_{X}\left(\mathrm{SMT}_{N}\right)$. Поэтому

$$
\frac{\rho_{X}\left(\mathrm{SMT}_{N_{i}}\right)}{\rho_{X}\left(\mathrm{MST}_{N_{i}}\right)} \leqslant \frac{\rho_{Y}\left(\mathrm{SMT}_{N^{\prime}}\right)+\varepsilon_{i}}{\rho_{Y}\left(\mathrm{MST}_{N^{\prime}}\right)}
$$

откуда, учитьвая, что $\left\{N_{i}\right\} \subset\left\{N: f(N)=N^{\prime}\right\}$, получаем

$$
\begin{aligned}
& \inf _{\left\{N^{\prime}: N^{\prime} \subset Y\right\}} \inf _{\left\{N: f(N)=N^{\prime}\right\}} \frac{\rho_{X}\left(\mathrm{SMT}_{N}\right)}{\rho_{X}\left(\mathrm{MST}_{N}\right)} \\
& \leqslant \inf _{\left\{N^{\prime}: N^{\prime} \subset Y\right\}} \inf _{\left\{N_{i}\right\}} \frac{\rho_{X}\left(\mathrm{SMT}_{N_{i}}\right)}{\rho_{X}\left(\mathrm{MST}_{N_{i}}\right)} \leqslant \inf _{\left\{N^{\prime}: N^{\prime} \subset Y\right\}} \inf _{i} \frac{\rho_{Y}\left(\mathrm{SMT}_{N^{\prime}}\right)+\varepsilon_{i}}{\rho_{Y}\left(\mathrm{MST}_{N^{\prime}}\right)} \\
& =\inf _{\left\{N^{\prime}: N^{\prime} \subset Y\right\}} \frac{\rho_{Y}\left(\mathrm{SMT}_{N^{\prime}}\right)}{\rho_{Y}\left(\mathrm{MST}_{N^{\prime}}\right)}=m\left(Y, \rho_{Y}\right) .
\end{aligned}
$$

Доказательство закончено.

Пусть $M$ - произвольное связное $n$-мерное риманово многообразие. Для каждой кусочно-гладкой кривой $\gamma$ обозначим через $\operatorname{len}(\gamma)$ ее длину по отношению к римановой метрике. Обозначим через $\rho$ внутреннюю метрику, порожденную римановой метрикой. Напомним, что

$$
\rho(x, y)=\inf _{\gamma} \operatorname{len}(\gamma),
$$

где точная нижняя грань берется по всем кусочно-гладким кривым $\gamma$, соединяюшим точки $x$ и $y$.

Пусть $P$ - некоторая точка из $M$. Рассмотрим нормальные координаты $x^{1}, \ldots, x^{n} \mathrm{c}$ центром в точке $P$, для которых риманова метрика $g_{i j}$, вычисленная в точке $P$, совпадает с $\delta_{i j}$. Пусть $U(\delta)$ - выпукльй открытьй шар радиуса $\delta$ с центром в $P$, для которого 
каждая его пара точек $x$ и $y$ соединяется единственной кратчайшей геодезической $\gamma$, лежащей в $U(\delta)$. При этом, $\rho(x, y)=\operatorname{len}(\gamma)$. Таким образом, шар $U(\delta)$ является метрическим пространством с внутренней метрикой, т.е. метрикой, в которой расстояние между точками равно точной нижней грани длин всех измеримых кривых, соединяющих эти точки. Отметим, что в координатах $x^{i}$ шар $U(\delta)$ задается так:

$$
U(\varepsilon)=\left\{\left(x^{1}\right)^{2}+\cdots+\left(x^{n}\right)^{2}<\delta^{2}\right\}
$$

поэтому если на $U(\delta)$ задать евклидово расстояние $\rho_{e}$ (по отношению к нормальным координатам $\left.x^{i}\right)$, то метрическое пространство $\left(U(\delta), \rho_{e}\right)$ также будет пространством с внутренней метрикой, порожденной евклидовой метрикой $\delta_{i j}$.

В силу гладкой зависимости метрики $g_{i j}(x)$ от точки $x \in U(\varepsilon)$ для любого $\varepsilon, 1 / n^{2}>$ $\varepsilon>0$, существует $\delta>0$ такое, что для всех точек $x \in U(\delta)$ выполняется

$$
\left|g_{i j}(x)-\delta_{i j}\right|<\varepsilon
$$

Последнее приводит к следующему предложению.

ПРЕДЛОЖЕНИЕ 2.4. Пусть $\|v\|_{g}$ обозначает длину касательного вектора $v \in T_{x} M$ в метрике $g_{i j}$, a $\|v\|_{e}-$ длину әтого вектора в евклидовой метрике $\delta_{i j}$. Если для любых $i$ и $ј$ выполняется неравенство (4), то

$$
\sqrt{1-n^{2} \varepsilon}\|v\|_{e} \leqslant\|v\|_{g} \leqslant \sqrt{1+n^{2} \varepsilon}\|v\|_{e} .
$$

ДокАЗАТЕЛЬСтво. Рассмотрим ортогональное (по отношению к $\delta_{i j}$ ) преобразование, приводящее $\left(g_{i j}\right)$ к диагональному виду $\operatorname{diag}\left(\lambda_{1}, \ldots, \lambda_{n}\right)$, и пусть $\left(c_{j}^{i}\right)$ - матрища этого преобразования. Тогда $\lambda_{k}=\sum_{i, j} c_{k}^{i} c_{k}^{j} g_{i j}$; поэтому, так как в силу ортогональности матрищы $\left(c_{j}^{i}\right)$ вьполняется $\left|c_{j}^{i}\right| \leqslant 1$, имеем

$$
\left|\lambda_{k}-1\right|=\left|\sum_{i, j}\left(c_{k}^{i} c_{k}^{j} g_{i j}-c_{k}^{i} c_{k}^{j} \delta_{i j}\right)\right| \leqslant \sum_{i, j}\left|c_{k}^{i}\right| \cdot\left|c_{k}^{j}\right| \cdot\left|g_{i j}-\delta_{i j}\right| \leqslant \sum_{i, j}\left|g_{i j}-\delta_{i j}\right| \leqslant n^{2} \varepsilon .
$$

В новых координатах имеем

$$
\|v\|_{g}=\sqrt{\sum_{k} l_{k} v^{k} v^{k}} \leqslant \sqrt{\max _{k} l_{k} \sum_{k} v^{k} v^{k}} \leqslant \sqrt{1+n^{2} \varepsilon}\|v\|_{e} .
$$

Аналогично получаем

$$
\|v\|_{g} \geqslant \sqrt{1-n^{2} \varepsilon}\|v\|_{e}
$$

Доказательство закончено.

Вспоминая определение расстояния между точками связного риманова многообразия, мгновенно получаем следуюший результат. 
СЛЕДСТВИЕ 2.1. Пусть $M$ - произвольное $n$-мерное риманово многообразие, а $U(\delta), \rho$ и $\rho_{e}$ такие, как выше. Тогда для любого $1 / n^{2}>\varepsilon>0$ существует $\delta>0$ такое, что для всех точек $x, y \in U(\delta)$ выполняется

$$
\sqrt{1-n^{2} \varepsilon} \rho_{e}(x, y) \leqslant \rho(x, y) \leqslant \sqrt{1+n^{2} \varepsilon} \rho_{e}(x, y) .
$$

Следствие 2.1, лемма 2.1 и независимость отношения Штейнера от выбора вьпуклого открытого подмножества в $\mathbb{R}^{n}$ приводят к следующему результату.

СлЕДСТВИЕ 2.2. Пусть $M$ - произвольное $n$-мерное риманово многообразие, $и$ $U(\varepsilon) \subset M$ - открытый выпуклый шар малого радиуса $\varepsilon$ с иентром в некоторой точке $P$. Обозначим через $\rho$ метрику на $M$, порожденную римановой метрикой. Тогда

$$
\sqrt{\frac{1-n^{2} \varepsilon}{1+n^{2} \varepsilon}} m\left(\mathbb{R}^{n}\right) \leqslant m(U(\varepsilon), \rho) \leqslant \sqrt{\frac{1+n^{2} \varepsilon}{1-n^{2} \varepsilon}} m\left(\mathbb{R}^{n}\right),
$$

где $m\left(\mathbb{R}^{n}\right)$ - отношение Штейнера для евклидова пространства $\mathbb{R}^{n}$.

Перейдем теперь к доказательствам основных теорем.

ДОКАЗАТЕЛЬСТВО ТЕОРЕМЫ 1.1 . Пусть $M$ - произвольное связное $n$-мерное риманово многообразие, и $\rho$ - метрика, порожденная римановой метрикой многообразия $M$. Пусть $P \in M$ - произвольная точка из $M$, и $U(\varepsilon)$ - открытьй вьпуклый шар радиуса $\varepsilon<1 / n^{2}$ с центром в $P$. Введем в $U(\varepsilon)$ нормальные координаты $x^{i}$, и пусть $\rho_{e}-$ метрика на $U(\varepsilon)$, порожденная евклидовой метрикой $\delta_{i j}$ (по отношению к координатам $x^{i}$ ).

Для некоторой убывающей последовательности $\varepsilon_{i}$ положительных чисел такой, что $\varepsilon_{i}<\varepsilon$ для любого $i$ и $\varepsilon_{i} \rightarrow 0$ при $i \rightarrow \infty$, рассмотрим семейство вложенных подмножеств $X_{i}=U\left(\varepsilon_{i}\right)$. Заметим, что в силу выпуклости евклидовых шаров $\left(U(\varepsilon), \rho_{e}\right)$ имеем

$$
m\left(U(\varepsilon), \rho_{e}\right)=m\left(\mathbb{R}^{n}\right)
$$

Кроме того, в силу вьпуклости шаров $U(\varepsilon)$ по отношению к внутренней метрике $\rho^{\prime}$, порожденной римановой метрикой $g_{i j}$, эта внутренняя метрика совпадает с ограничением метрики $\rho$. Таким образом, шар $U(\varepsilon)$ с внутренней метрикой $\rho^{\prime}$ является подпространством в $(M, \rho)$.

По следствию 2.2

$$
m\left(X_{i}, \rho\right) \leqslant \sqrt{\frac{1+n^{2} \varepsilon}{1-n^{2} \varepsilon}} m\left(\mathbb{R}^{n}\right) .
$$

Так как $\sqrt{\left(1+n^{2} \varepsilon\right) /\left(1-n^{2} \varepsilon\right)} \rightarrow 1$ при $i \rightarrow \infty$ в силу выбора последовательности $\varepsilon_{i}$, получаем

$$
\inf _{i} m\left(X_{i}, \rho\right) \leqslant m\left(\mathbb{R}^{n}\right)
$$

но по лемме 2.2

$$
m(M, \rho) \leqslant \inf _{i} m\left(X_{i}, \rho\right),
$$

что и завершает доказательство теоремы. 
ДОКАЗАТЕЛЬСТВО ТЕОРЕМЫ 1.2. Пусть $\pi: W \rightarrow M-$ локально изометричное накрытие, где $W$ и $M$ - связные римановы многообразия. Обозначим через $\rho_{W}$ и $\rho_{M}$ метрики, порожденные соответствующими римановыми метриками на $W$ и $M$. Отметим, что локально изометричное накрытие не увеличивает расстояний, так как образ измеримой кривой $\gamma$ имеет ту же длину, что и $\gamma$.

Рассмотрим произвольное конечное множество $N^{\prime} \subset M$, и пусть $G_{i}^{\prime}$ - семейство деревьев на конечных множествах $\bar{N}_{i}^{\prime} \supset N^{\prime}$ таких, что

$$
\rho_{M}\left(G_{i}^{\prime}\right) \rightarrow \rho_{M}\left(\mathrm{SMT}_{N^{\prime}}\right) \quad \text { при } i \rightarrow \infty .
$$

Для каждого $G_{i}^{\prime}$ обозначим через $\Gamma_{i}^{\prime}$ вложенную сеть на $M$ типа $G_{i}^{\prime}$ с множеством вершин $V\left(G_{i}^{\prime}\right)$, длина которой отличается от $\rho_{M}\left(G_{i}^{\prime}\right)$ не более, чем на $1 / i$. Пусть $\Gamma_{i}-$ связная компонента из $\pi^{-1}\left(\Gamma_{i}^{\prime}\right)$ и $N_{i}=\pi^{-1}(N) \cap \Gamma_{i}$. Так как сеть $\Gamma_{i}^{\prime}$ стягиваема, ограничение расслоения $\pi$ на $\Gamma_{i}^{\prime}$ тривиально, поэтому ограничение проекции $\pi$ на $\Gamma_{i}$ является гомеоморфизмом. В силу локальной изометричности проекции $\pi$ получаем, что длина сети $\Gamma_{i}$ на $W$ совпадает с длиной сети $\Gamma_{i}^{\prime}$ на $M$. Но $\rho_{W}\left(\mathrm{SMT}_{N_{i}}\right)$ не превосходит длины сети $\Gamma_{i}$, поэтому

$$
\rho_{W}\left(\mathrm{SMT}_{N_{i}}\right) \leqslant \rho_{M}\left(\mathrm{SMT}_{N^{\prime}}\right)+\varepsilon_{i}
$$

где положительные числа $\varepsilon_{i}$ стремятся к 0 при $i \rightarrow \infty$. Поэтому

$$
\inf _{\left\{N: f(N)=N^{\prime}\right\}} \rho_{W}\left(\mathrm{SMT}_{N}\right) \leqslant \rho_{M}\left(\mathrm{SMT}_{N^{\prime}}\right) .
$$

Осталось применить предложение 2.3. Доказательство закончено.

ДокАЗАТЕЛЬСТВо СЛЕДСТВИЯ 1.1 вытекает из теорем 1.1 и 1.2.

ДокАЗАТЕЛЬСТво С ЕдСТВИя 1.2 вытекает из следствия 1.1, теоремы Ду и Хванга [1], утверждающей, что отношение Штейнера для евклидовой плоскости равно $\sqrt{3} / 2$, а также теоремы Рубинштейна и Венга [4], утверждающей, что отношение Штейнера двумерной сферы с метрикой постоянной кривизны равно $\sqrt{3} / 2$.

ДокАЗАТЕЛЬСтво тЕОРЕмЫ 1.3. Рассмотрим модель Пуанкаре для пространства Лобачевского $L^{2}(-1)$ постоянной кривизны - 1 . Напомним, что эта модель представляет собой плоский круг радиуса 1 с центром в нуле на евклидовой плоскости с координатами $(x, y)$, причем метрика $d s^{2}$ в нем задается в виде

$$
d s^{2}=4 \frac{d x^{2}+d y^{2}}{\left(1-x^{2}-y^{2}\right)^{2}}
$$

Известно, что каждьй правильный треугольник на плоскости Лобачевского можно описать окружностью, причем радиусы, проведенные из центра окружности в вершины треугольника, образуют углы величины $120^{\circ}$.

Пусть $r$ - радиус описанной окружности. По теореме косинусов длина $a$ стороны правильного треугольника вычисляется по формуле

$$
\operatorname{ch} a=\operatorname{ch}^{2} r-\operatorname{sh}^{2} r \cos \frac{2 \pi}{3}=1+\frac{3}{2} \operatorname{sh}^{2} r .
$$


Легко проверить, что длина MST для такого треугольника равна $2 a$, а длина SMT равна $3 r$. Поэтому отношение Штейнера $m(r)$ для правильного треугольника на $L^{2}(-1)$, вокруг которого можно описать окружность радиуса $r$, имеет вид

$$
m(r)=\frac{3}{2} \cdot \frac{r}{\operatorname{arcch}\left(1+\frac{3}{2} \operatorname{sh}^{2}(r)\right)}
$$

Легко вычислить, что предел функции $m(r)$ при $r \rightarrow \infty$ равен $3 / 4$. Доказательство закончено.

ДОКАЗАТЕЛЬСТВО ТЕОРЕМЫ 1.4 вытекает из того, что функция $m(r)$ в окрестности нуля имеет следующий начальный отрезок ряда Тейлора:

$$
\frac{\sqrt{3}}{2}-\frac{r^{2}}{16 \sqrt{3}}+O\left(r^{4}\right),
$$

поэтому в некоторой окрестности $(0, \varepsilon)$ она строго меньше $\sqrt{3} / 2$. Последнее означает, что у достаточно малых правильных треугольников на поверхности постоянной кривизны -1 отношение длин SMT и MST строго меньше $\sqrt{3} / 2$.

\section{СПИСОК ЦИТИРОВАННОЙ ЛИТЕРАТУРЫ}

[1] Du D. Z., Hwang F. K. A proof of Gilbert-Pollak Conjecture on the Steiner ratio // Algorithmica. 1992. V. 7. P. 121-135.

[2] Gilbert E. N., Pollak H. O. Steiner minimal trees // SIAM J. Appl. Math. 1968. V. 16. № 1. P. 1-29.

[3] Cieslik D. Steiner Minimal Trees. Kluwer Academic Publishers, 1998.

[4] Rubinstein J. H., Weng J. F. Compression theorems and Steiner ratios on spheres // J. Combin. Optimization. 1997. V. 1. P. 67-78.

[5] Ivanov A. O., Tuzhilin A. A. Minimal Networks. The Steiner Problem and Its Generalizations. N.W., Boca Raton, Florida: CRC Press, 1994.

[6] Ivanov A. O., Tuzhilin A. A. Branching Solutions of One-Dimensional Variational Problems. World Publisher Press, 2000, to appear.

[7] Hwang F. K., Richards D., Winter P. The Steiners Tree Problem. Elsevier Science Publishers, 1992.

(А. О. Иванов, А. А. Тужилин) Московский государственньй

Поступило университет им. М.В. Ломоносова 10.04 .2000

(Д. Цислик) Институт математики и информатики,

Эрнст Морис Арндт университет,

г. Грайфсвальд, Германия

E-mail :

aoiva@mech.math.msu.su

tuz@mech .math .msu.su

cieslik@mail.uni-greifswald.de 